\title{
CSF Neurofilament Proteins Levels are Elevated in Sporadic Creutzfeldt-Jakob Disease
}

\author{
Jeroen J.J. van Eijk ${ }^{\mathrm{a}}$, Bart van Everbroeck ${ }^{\mathrm{b}}$, W. Farid Abdo ${ }^{\mathrm{a}}$, Berry P.H. Kremer ${ }^{\mathrm{a}, \mathrm{c}}$ and \\ Marcel M. Verbeek ${ }^{\mathrm{a}, \mathrm{d}, *}$ \\ ${ }^{a}$ Department of Neurology, Radboud University Nijmegen Medical Centre, Donders Institute for Brain, Cognition \\ and Behaviour, Alzheimer Centre Nijmegen, The Netherlands \\ ${ }^{\mathrm{b}}$ Biobank, Born Bunge Institute, University of Antwerp, Wilrijk, Belgium \\ ${ }^{\mathrm{c}}$ Department of Neurology, University Medical Centre Groningen, The Netherlands \\ ${ }^{\mathrm{d}}$ Department of Laboratory Medicine, Radboud University Nijmegen Medical Centre, The Netherlands
}

Handling Associate Editor: Nigel Hooper

Accepted 26 March 2010

\begin{abstract}
In this study we investigated the cerebrospinal fluid (CSF) levels of neurofilament light (NFL) and heavy chain (NFHp35), total tau (t-tau), and glial fibrillary acidic protein (GFAP) to detect disease specific profiles in sporadic Creutzfeldt Jakob disease (sCJD) patients and Alzheimer's disease (AD) patients. CSF levels of NFL, NFHp35, t-tau, and GFAP of 23 sCJD patients and $55 \mathrm{AD}$ patients were analyzed and compared to non-demented controls. Median NFL, NFHp35, GFAP, and $\mathrm{t}$-tau levels were significantly increased in SCJD patients and AD patients versus controls $(p<0.0001$ in all). NFL, NFHp35, and t-tau levels were significantly increased in SCJD patients versus AD patients $(p<0.005)$, but GFAP concentrations did not differ between sCJD and AD. The results suggest that neuroaxonal damage, reflected by higher CSF levels of NFL, NFHp35, and $\mathrm{t}$-tau, is more pronounced in the pathophysiology of SCJD than in AD. The comparable CSF GFAP concentrations suggest that astroglial damage or astrocytosis is equally pronounced in the pathophysiology of AD and SCJD. Prospective studies are needed to determine whether NFL and NFHp35 may be additional tools in the differential diagnosis of rapidly progressive dementias.
\end{abstract}

Keywords: Alzheimer's disease, cerebrospinal fluid, Creutzfeldt-Jakob disease, diagnosis, prion disease

\section{INTRODUCTION}

Alzheimer's disease (AD) is a devastating neurodegenerative disorder characterized by widespread loss of cortical neurons and their connections resulting in memory loss and cognitive decline. Sporadic

\footnotetext{
*Correspondence to: Dr. Marcel M. Verbeek, Radboud University Nijmegen Medical Centre, Donders Institute for Brain, Cognition and Behaviour, 830 LGEM, Neurochemistry Lab, P.O. Box 9101, 6500 HB Nijmegen, The Netherlands. Tel.: +31 24 3615192; Fax: +31 24 3668754; E-mail: m.verbeek@neuro.umcn.nl.
}

Creutzfeldt-Jakob disease (sCJD) is a rare, fatal neurodegenerative disorder, belonging to the spongiform encephalopathies which is clinically characterized by a rapidly progressive dementia with visual or cerebellar signs, (extra)pyramidal symptoms, and ultimately akinetic mutism.

In $\mathrm{AD}$, the typical cerebrospinal fluid (CSF) pattern includes elevated total tau (t-tau) and hyperphosphorylated tau (p-tau) proteins, whereas the amyloid- $\beta_{42}$ protein $\left(\mathrm{A} \beta_{42}\right)$ concentration is decreased [1]. In SCJD, detection of 14-3-3 protein is highly sensitive and is part of the WHO diagnostic criteria for SCJD [2], and 
strongly elevated levels of t-tau protein are highly specific. Other abnormal proteins in CSF of SCJD patients include elevated S100B and neuron-specific enolase (NSE) and decreased $\mathrm{A} \beta_{42}[3,4]$. However, CSF $\mathrm{t}$-tau levels are usually much higher in SCJD than in AD and $\mathrm{A} \beta_{42}$ levels are usually much lower in $\mathrm{AD}$ than in sCJD.

Neurofilament (NF) proteins are neuronal cytoskeleton proteins. They play an important role in neuronal structure and are mainly located in large myelinated neurons. NF-proteins are highly phosphorylated and are composed of 3 subunits defined by their molecular weight: $68 \mathrm{kDa}$ (NF-light chain, NFL), $160 \mathrm{kDa}$ (NFmedium chain, NFM), and $200 \mathrm{kDa}$ (NF-heavy chain, $\mathrm{NFH}$ ). Elevated CSF levels of NF-proteins have been found in vascular dementia, $\mathrm{AD}$, and frontotemporal dementia (FTD) and it was demonstrated that CSF NFL analysis may help to distinguish FTD from AD [5-7]. $\mathrm{NF}$-protein analysis in SCJD has not been reported so far, although neuropathological reports on neurofilament inclusions in SCJD suggest neurofilament levels could be increased in SCJD [8]. In addition, since the concentration of the glial protein $\mathrm{S} 100 \mathrm{~B}$ is significantly increased in CSF of SCJD patients and because of the reactive astrocytosis in SCJD, it can be speculated that concentrations of the astrocyte marker glial fibrillary acidic protein (GFAP) will also be increased in CSF of SCJD patients.

The aim of the present study was to identify whether markers of neuronal damage (NFL, NFHp35, t-tau) and glial damage (GFAP) are abnormal in CSF of sCJD and $\mathrm{AD}$ patients and to determine a disease specific profile.

\section{MATERIALS AND METHODS}

\section{Patients}

This retrospective study included 23 sCJD patients and $55 \mathrm{AD}$ patients identified through the CSF databases of the Radboud University Nijmegen Medical Centre and the Born Bunge Institute (University of Antwerp) between January 1998 and January 2005. Inclusion into the study was dependent upon the availability of CSF for additional analyses and of the availability of sufficient clinical information to make a proper diagnosis. Only patients with a probable diagnosis according to standard diagnostic criteria were included. The SCJD patients fulfilled the WHO criteria for probable SCJD and a diagnosis of probable $\mathrm{AD}$ was made according to the revised NINCDS-ADRDA criteria [2,9]. The diagnostic examination included physical, neurological and mental status examination, screening laboratory tests, routine CSF analysis, and MRI or CT of the brain. The sCJD patients also underwent EEG. Twenty probable sCJD patients had converted to definite SCJD by the time of data analysis. In the remaining 3 probable sCJD patients, postmortem neuropathological examination had not been allowed by the family. Two of them were tested for 14-3-3 and were positive; the other one had not been analyzed for 14-3-3, but had a typical EEG (with periodic sharp wave complexes) and died after a total disease duration of 14 months. None of the sCJD patients survived more than 2 years after having been diagnosed with SCJD. The SCJD patients from the Belgian center $(n=15)$ were also included in a previous study [10]; the other SCJD patients were not published previously.

The study contained some missing data, since the archived CSF was not sufficient for all analyses. The sCJD patients $(n=23)$ had all been tested for NFHp35 and t-tau, whereas 21 patients had been tested for NFL or $14-3-3$ and 18 for GFAP. All AD patients $(n=55)$ had been tested for NFL and t-tau, whereas 52 had been tested for NFHp35, 51 for GFAP, and 40 for 14-3-3. Disease duration at the time of lumbar puncture was also recorded and available in all patients.

For determination of CSF biomarker concentration in a control population, non-demented controls were included with ages between $50-85$ years who were referred to the Department of Neurology of the RUNMC and underwent a lumbar puncture in a diagnostic workup, but who, after extensive examination, turned out not to have a neurological disease, and who had normal CSF cell count, hemoglobin, bilirubin, total protein, lactate, glucose and no oligoclonal IgG bands. For NFHp35, 21 controls (mean age 57 yr, SD 7.9) were used. For NFL, 23 controls (mean age 57.6 yr, SD 7.9) were used, for t-tau 80 controls (mean age $60.2 \mathrm{yr}$, SD 8.8), and for GFAP 31 controls (mean age $58.7 \mathrm{yr}$, SD 8.4) were used.

\section{CSF analysis}

CSF was obtained by lumbar puncture, collected in polypropylene tubes, and transferred to the hospital laboratory within $30 \mathrm{~min}$. Lumbar punctures were performed after informed consent was obtained from the patient, or from his or her legal representative. After routine investigations, CSF was centrifuged (860 g, $5 \mathrm{~min}$ ), aliquoted and stored at $-80^{\circ} \mathrm{C}$ until analysis. The concentrations of $\mathrm{t}$-tau and GFAP were analyzed 
Table 1

Patient characteristics and concentrations of CSF biomarkers

\begin{tabular}{|c|c|c|c|c|}
\hline & $\mathrm{sCJD}$ & $\mathrm{AD}$ & CONT & p-value \\
\hline Number of patients & 23 & 55 & 130 & \\
\hline Gender $(\mathrm{m} / \mathrm{f})$ & $10 / 13$ & $23 / 32$ & & N.S. ${ }^{1}$ \\
\hline *Age (yrs) & $68.4(8.2)$ & $68.7(8.8)$ & $59.2(8.4)$ & N.S. ${ }^{1} ;<0.0001^{2,3}$ \\
\hline \# Duration (months) & $5(2-9.5)$ & $24(18-40)$ & N.A. & $<0.0001^{1}$ \\
\hline *MMSE & N.A. & $19(5.5)$ & N.A. & N.A. \\
\hline \# NFHp35 (ng/L) & $579(420-796)$ & $117(77-165)$ & $75(61-88)(n=21)$ & $<0.0001^{1,2} ; 0.0007^{3}$ \\
\hline${ }^{\#} \mathrm{NFL}(\mathrm{ng} / \mathrm{L})$ & $78.0(32.6-123.3)$ & $7.2(6.0-18.1)$ & $5.20(0-19.7)(n=23)$ & $<0.0001^{1,2} ; 0.044^{3}$ \\
\hline${ }^{*}$ GFAP $(\mu \mathrm{g} / \mathrm{L})$ & $2.4(1.3-6.1)$ & $2.9(2.3-3.7)$ & $1.39(0.6-2.2)(n=31)$ & $0.054^{1} ; 0.0037^{2} ;<0.0001^{3}$ \\
\hline \# Tau (ng/l) & $6438(2400-15230)$ & $593(388-795)$ & $179(136-253)(n=80)$ & $<0.0001^{1,2,3}$ \\
\hline$\$ 14-3-3$ & $20(n=21)$ & $5(n=40)$ & N.A. & $<0.0001^{1}$ \\
\hline
\end{tabular}

Abbreviations: sCJD, sporadic Creutzfeldt-Jakob disease; AD, Alzheimer's disease; CONT, non-demented controls; NFL, neurofilament light chain; NFHp35, phosphorylated neurofilament heavy chain; GFAP, glial fibrillary acidic protein; Tau, tau-protein.

\# Median (25th-75th percentile) are shown. ${ }^{*}$ Mean (standard deviation) are shown. ${ }^{\$}$ Number of positive tests is shown (total number of tests). N.S., not significant; N.A., not applicable, n: number. ${ }^{1}$ sCJD compared to AD, ${ }^{2}$ sCJD compared to CONT,

${ }^{3} \mathrm{AD}$ compared to CONT. p-values for NFHp35 and NFL; Mann Whitney-tests; p-values for GFAP and t-tau: t-tests.

routinely every two weeks, i.e., at a maximum storage time at $-80^{\circ} \mathrm{C}$ of two weeks after sample collection. GFAP concentrations were analyzed by a homemade sandwich ELISA as described earlier [11]. The interassay CV\% is $8.6 \%$ ( $n=16$; at a concentration of $4.5 \mu \mathrm{g} / \mathrm{L}$ ). T-tau concentrations in CSF were analyzed by using the Innotest hTau assay (Innogenetics, Ghent, Belgium). The inter-assay CV\% is $6.9 \%(n=20$; at a concentration of $263 \mathrm{ng} / \mathrm{L}$ ). Previously described sandwich ELISAs were used to determine NFL and NFHp35 levels $[12,13]$. Both NFL and NFHp35 were retrospectively analyzed in the patient groups after the selection of the patient groups was completed. The inter-assay $\mathrm{CV}$ for NFL was $3.9 \%$ at a concentration of $124 \mathrm{ng} / \mathrm{l}$ $(n=8), 9.1 \%$ at a concentration of $60 \mathrm{ng} / \mathrm{l}(n=14)$, and $17.8 \%$ at a concentration of $26 \mathrm{ng} / \mathrm{l}(n=15)$. For $\mathrm{NFHp} 35$, the interassay CV was $18 \%$ at a concentration of $35 \mathrm{ng} / \mathrm{L}(n=18)$. The presence of the 14-3-3 protein in CSF was routinely determined every two weeks by electrophoresis and immunoblotting. In brief, CSF proteins were separated by SDS-PAGE electrophoresis. Proteins were transferred to nitrocellulose membranes, after which the membranes were successively incubated overnight with Odyssey blocking buffer (LI-COR Biosciences, Lincoln, NE, USA), anti-14-3-3 $\beta$ antibody (Santa Cruz Biotech, 2 h) and Alexa Fluor680labeled goat anti-rabbit antibodies $(1 \mathrm{~h})$. Incubation steps were each followed by extensive washing in PBS. Blots were scanned by using Odyssey infrared imaging system (LI-COR Biosciences), according to manufacturer's instructions. Total protein concentrations were measured by using the Lowry method.

\section{Statistical analysis}

Kolmogorov-Smirnov normality tests showed nonGaussian distribution of CSF NFHp35 in the AD and control groups and of NFL in the AD group. Therefore, data of NFHp35, NFL, t-tau, and GFAP are all presented as median and p25-p75 range, and nonparametric Mann-Whitney tests were used to analyze non-Gaussian distributed CSF data, whereas t-tests were used to analyze Gaussian distributed CSF data. Adjustments for age, gender, disease duration and total protein concentrations were performed by using linear regression. Correlations between CSF and clinical parameters were analyzed using the Spearman test for non-Gaussian distributed variables and the Pearson test for Gaussian distributed variables; in case of CSF proteins a partial correlation analysis (by group) was used. Graphpad Prism (San Diego, CA) and SPSS16.0 software were used for statistical analysis.

\section{RESULTS}

Baseline characteristics and biochemical analyses are summarized in Table 1. All sCJD patients clinically suffered from a (severe) dementia syndrome. Abnormal triphasic waves on EEG analysis were observed in 14 patients, absent in 8 patients and could not be unequivocally determined in one patient. Myoclonic jerks were observed in 15 patients. Seven sCJD patients had pyramidal symptoms (spasticity and extensor plantar responses), 9 patients had extrapyramidal symptoms (hypo/ bradykinesia, chorea or rigidity), 16 patients had a cerebellar syndrome (ataxia) and in only 


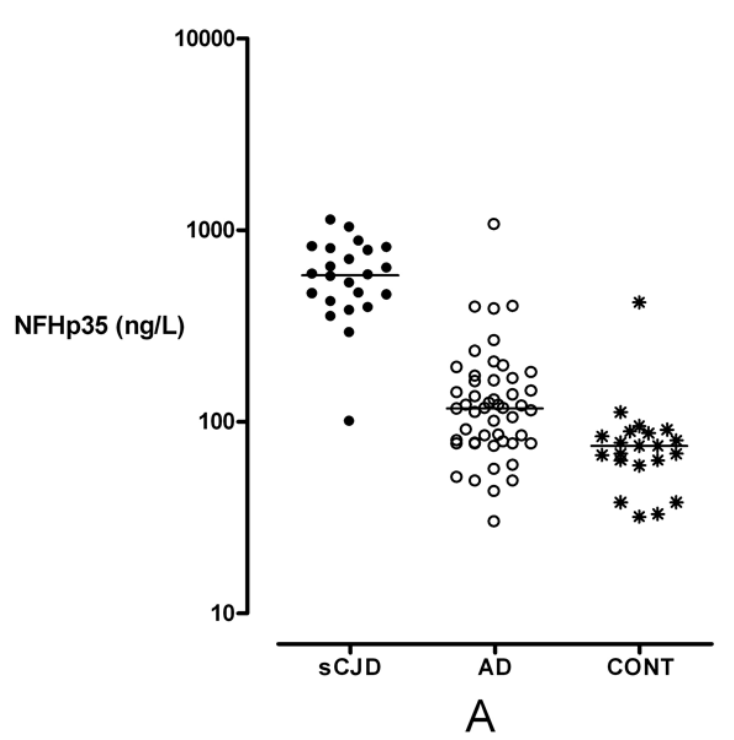

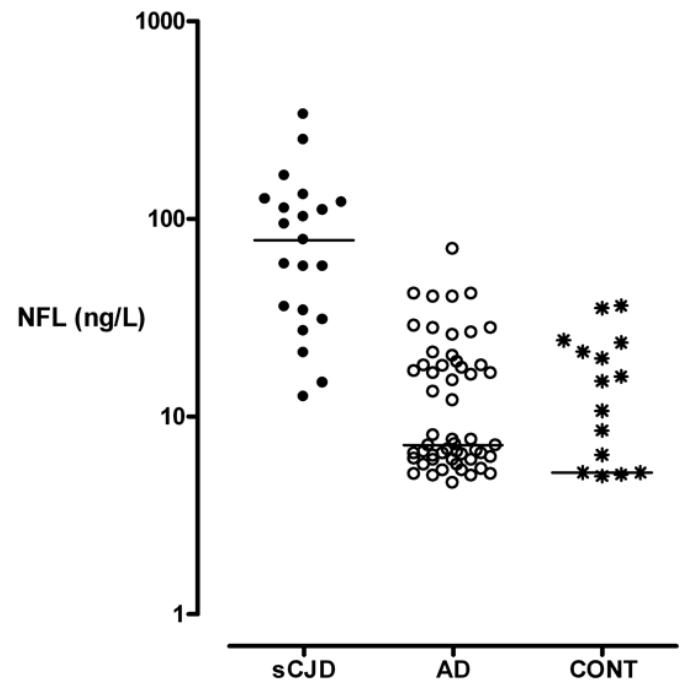

B

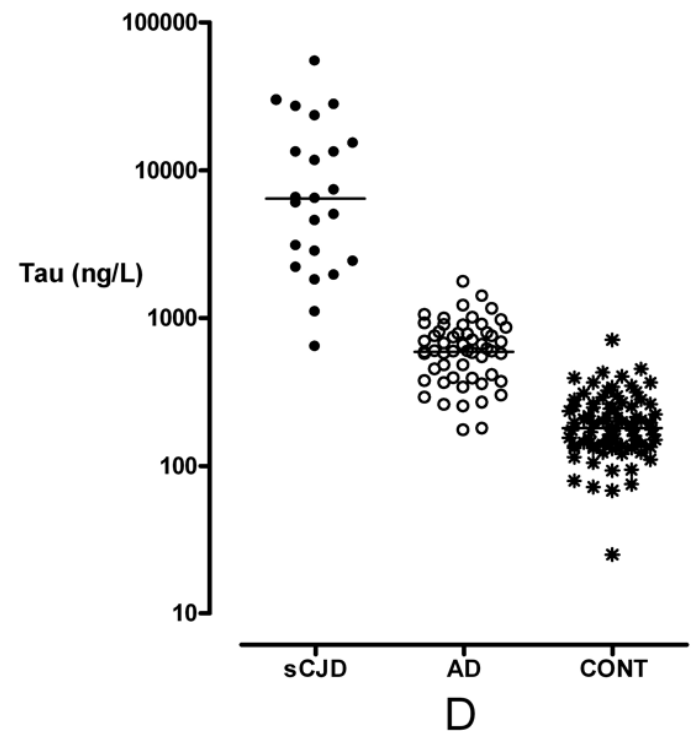

Fig. 1. Concentrations of NFHp35 (A), NFL (B), GFAP (C), and tau (D) per patient group. Horizontal lines indicate median levels. Abbreviations: sCJD, sporadic Creutzfeldt-Jakob disease; AD, Alzheimer's disease; CONT, controls.

7 sCJD patients typical abnormalities on MRI, e.g., bilateral hyper intense signal on $\mathrm{T} 2$ weighted images predominantly affecting the caudate nucleus and putamen, were observed.

Levels of CSF NFL, NFHp35, and t-tau were significantly increased in SCJD versus controls and SCJD versus AD patients (Table 1; Fig. 1a, b, d), whereas total protein concentrations were similar in $\mathrm{AD}$ (mean $513 \mathrm{mg} / \mathrm{L}$ ) and sCJD (mean $495 \mathrm{mg} / \mathrm{L} ; p=0.75$ ). Similar results were obtained after adjustment of the data for age, gender, disease duration and total protein concentrations. Also, similar p-values were obtained if we log-transformed the results for NFL, NFHp35, t-tau, and GFAP (to normalize the data distribution for NFL and NFHp35), either with or without adjustment for age, gender, and disease duration. GFAP levels were higher in either SCJD or AD versus controls (Table 1; Fig. 1c), but did not differ significantly between sCJD and AD patients (Table 1; Fig. 1c). The differences in GFAP results between AD and controls, but not between SCJD and controls, remained significant after adjustment for age, gender, and disease duration. Furthermore, the differences between SCJD and AD remained significant in those subgroups ( $\mathrm{SCJD}, n=17$; 
$\mathrm{AD}, n=44$ ) for whom all CSF data of NFL, NFHp35, t-tau, and GFAP were available. The test for the presence of the 14-3-3 protein in CSF by immunoblotting was positive in 20 cases with SCJD and 5 cases with $\operatorname{AD}(p<0.0001)$.

No significant correlation between CSF markers and either age or disease duration was found in SCJD patients. In $\mathrm{AD}$ patients, we found a weak correlation between NFL concentration and age $(r=0.297 ; p=$ 0.022 ), but not with disease duration. No other correlations were found between the various CSF markers and clinical parameters in either SCJD or AD patients. Correlations between the CSF proteins are shown in Table 3; significant correlations were observed between NFH, NFL, and GFAP, between GFAP and t-tau, but not between $\mathrm{t}$-tau and either NFL or NFH.

The results of the ROC analysis to discriminate AD from sCJD are shown in Table 2. NFL, NFHp35 and tau analysis all resulted in a high area under the ROC curve and high likelihood ratio. Binary (multivariate) logistic regression analysis selected NFL, NFHp35, tau and duration as independent contributors for differentiation between SCJD and AD according to the model: $\mathrm{Y}=$ 33.68-0.465* NFL-0.15* NFHp35-0.026* tau + $1.98 *$ duration (months). The ROC area under curve of this equation was 0.998 and therefore almost perfectly discriminated SCJD from AD patients.

\section{DISCUSSION}

In this study, we found significantly elevated concentrations in CSF of NFHp35, NFL, t-tau, and GFAP in SCJD and AD compared to controls. Concentrations of CSF NFL, NFHp35, and t-tau were also significantly elevated in SCJD compared to AD patients, but GFAP levels did not differ between these two patient groups.

Both human and animal studies have shown that NFproteins play an important role in the axonal environment. These highly phosphorylated proteins are essential in maintaining neuronal cytoskeletal plasticity by influencing axonal caliber and axonal transport [14]. Neuropathological examination in sCJD has shown numerous abnormal neurites labeled by NF-protein immunocytochemistry [8]. In previous studies it has been shown that elevated NFL levels are associated with axonal and white matter damage in neurological diseases such as multiple sclerosis, FTD, and multiple system atrophy $[7,13,15]$. NF-proteins may be regarded as biomarkers for degeneration of large, myelinated axons. Our data suggest that these axons are either more subject to degeneration in SCJD than $\mathrm{AD}$ or release NF-proteins during the course of this disease, which may lead to the highly elevated CSF concentrations. A close observation of the NFL data in the AD group revealed a potential bimodal distribution of the data; we did not, however, find a correlation with sampling date or storage time which may explain this difference.

T-tau concentration was also strongly elevated in SCJD relative to AD. T-tau is a microtubule associated protein mainly localized in neuronal axons where it has a function in maintaining the normal cytoskeleton, by stabilizing microtubules that are essential for axonal transport. Thus, the high concentration of t-tau in CSF of sCJD patients also suggests a more compromised axonal function in this disorder. Our data are in line with previous studies, in which strongly elevated t-tau concentrations were reported in CSF of sCJD patients [4, 10,16]. The concentrations of both NFL and t-tau were increased by a factor 10 in SCJD compared to AD, suggesting, at first sight, that similar pathophysiological mechanisms may lead to release of these axonal proteins into the CSF of SCJD patients. However, we did not find a correlation between NFL and t-tau levels, suggesting that independent mechanism contribute to the release of multiple axonal proteins in the extracellular space and, eventually, CSF. Furthermore, in previous studies we found that NFL and t-tau independently contributed to the differentiation of MSA from idiopathic Parkinson's disease [13]. Thus, although both NF and $\mathrm{t}$-tau proteins are both located in axons, these observations strongly suggest that different pathophysiological mechanisms lead to the release of these proteins into the extracellular space. This hypothesis is strengthened by the observation that the concentrations of NFHp35 in SCJD were elevated by a factor 5 compared to AD and also did not correlate to t-tau levels. The respective mechanisms that lead to these differences remain to be elucidated.

We did not find a significant difference in CSF GFAP concentration between SCJD and AD and the GFAP concentration in either group was only marginally, albeit significantly, increased compared to controls. GFAP is a cytoskeletal protein of astrocytes which is important for astrocyte motility and shape. GFAP expression is increased during reactive astrocytosis, a process that is associated with senile plaque formation in $\mathrm{AD}$ brains and that is a common feature of SCJD as well $[17,18]$. In a previous study, it was described that CSF GFAP concentrations were higher in AD than in sCJD [19]. Our data do not support this observation, but suggests that the degree of astrocytosis is compa- 
Table 2

ROC analysis of the CSF biomarkers NFL, NFHp35, t-tau, and 14-3-3

\begin{tabular}{lccccccc}
\hline CSF parameter & Cut-off value & Sens \% & Spec \% & PPV \% & NPV \% & Likelihood ratio & AUC (95\% CI) \\
\hline NFL (ng/l) & $>29.8$ & 81 & 91 & 77 & 93 & 4.8 & $0.93(0.87-0.99)$ \\
NFHp35 (ng/l) & $>276.5$ & 90 & 96 & 82 & 98 & 20.8 & $0.95(0.88-1.0)$ \\
t-tau (ng/l) & $>1072$ & 93 & 96 & 85 & 98 & 21.7 & $0.98(0.94-1.0)$ \\
$14-3-3$ & N.A. & 95 & 88 & 80 & 97 & 7.9 & $0.91(0.83-0.99)$ \\
\hline
\end{tabular}

Abbreviations: NFL, neurofilament light chain; NFHp35, phosphorylated neurofilament heavy chain; t-tau, total tau protein; Sens, sensitivity; Spec, Specificity; PPV, positive predictive value; NPV, Negative predictive value; AUC, area under curve. N.A., not applicable.

Table 3

Partial correlation analysis of the CSF proteins NFH, NFL, GFAP, and t-tau

\begin{tabular}{llll}
\hline & NFH & NFL & t-tau \\
\hline NFL & $R=0.473$ & & \\
& $p<0.0001$ & & \\
t-tau & $R=0.189$ & $R=0.077$ & \\
& $p=0.147$ & $p=0.558$ & \\
GFAP & $R=0.350$ & $R=0.449$ & $R=0.684$ \\
& $p=0.006$ & $p<0.0001$ & $p<0.0001$ \\
\hline
\end{tabular}

$R=$ partial regression coefficient.

rable in SCJD and AD. Interestingly, our correlation analysis demonstrated a correlation between t-tau and GFAP, suggesting that reactive astrocytosis is linked to axonal degeneration. However, given the magnitude of increase in CSF biomarkers, our observations showed that in particular neuro-axonal degeneration contributes to the pathophysiological mechanism of $\mathrm{SCJD}$, rather than astrocyte degeneration. GFAP expression is limited to astrocytes, whereas the protein $\mathrm{S} 100 \mathrm{~B}$ is a more general marker for the glial cell population in the brain, also including oligodendrocytes and Schwann cells.

It has been demonstrated previously that the CSF concentrations of S100B are increased in a majority of the sCJD patients, but is less pronounced in $\mathrm{AD}$ [3]. Therefore, it seems likely that degeneration of oligodendrocytes or Schwann cells, rather than that of astrocytes, contributes to the elevated S100B concentration in SCJD CSF.

The typical findings in CSF of SCJD patients can be summarized by elevated concentrations of the proteins 14-3-3, t-tau, NSE and S100B. In particular, the diagnostic accuracy of the testing for the presence in CSF of the 14-3-3 protein, as part of the WHO diagnostic criteria, has been extensively studied. Reported sensitivities to detect SCJD on the basis of 14-3-3 analysis vary between $83 \%$ and $100 \%$ with a specificity of $67 \%-100 \%$ [16,20-28]. Our data fit in with these numbers. A recent autopsy-based study on 280 consecutive cases clinically suspected of sCJD in the Netherlands, however, demonstrated a sensitivity of only $74 \%$ and specificity of $79 \%$, demonstrating the limitations of this biochemical assay [29].
Sensitivity for t-tau varied between $86 \%$ and $98 \%$ with a specificity of 75-97\% [16,20-22,25]. Sensitivity to detect SCJD is generally lower for NSE (79\%$85 \%$ ), with specificities ranging from $85 \%-92 \%$ [22, 23,28]. Finally, sensitivity to detect sCJD by using S100B quantification in CSF varied between $84 \%$ and $98 \%$ and specificity widely ranges from $29 \%$ to $90 \%$ [3, $23,25]$. In the light of these findings the combined sensitivity and specificity we report here for t-tau, NFL and NFHp35 are very well comparable with other biomarkers. In the case of t-tau NFHp35 these numbers are at the high end of reported ranges as are the likelihood ratios and area under the curves.

It should be noted, however, that the timing of the lumbar puncture relative to the onset of clinical symptoms may affect the diagnostic accuracy of several biomarkers. It has been reported that the sensitivity for 14-3-3, t-tau, and S100B is higher in patients with a lumbar puncture taken within 6 weeks after the clinical symptoms compared to a delayed lumbar puncture [25]. Contrasting reports have also been published, however [30]. Finally, the M/V polymorphism at position in the PRNP gene may also slightly affect the diagnostic accuracy of the respective biomarkers [25, 30]. One of the limitations of our study is its retrospective, unblinded nature which might have confounded the results by indication. The missing values might have introduced skewing of data as well; however, given the major differences we observed in the concentrations of CSF NFL, NFHp35, and t-tau, this effect will be of minor importance. In conclusion, we found a disease specific CSF profile of SCJD in which the very high concentrations of NFL, NFHp35, and t-tau reflect a more important role of neuroaxonal damage in SCJD compared to AD. In contrast, astroglial damage seems to play an equally important role in either SCJD or AD. Moreover, this study is the first to evaluate analysis of NF proteins in SCJD-patients. It demonstrated that levels of NFL and NFHp35 are significantly and strongly increased in SCJD versus AD and controls. The ability to measure the neurofilament levels quantitatively with 
an ELISA makes this analysis potentially interesting for diagnostic purposes and has advantages over the qualitative assessment of 14-3-3 protein in CSF, which is performed by immunoblotting. Assessment of the potential of NF protein analysis as an additional diagnostic value in rapidly progressive dementias awaits future prospective studies.

\section{ACKNOWLEDGMENTS}

The authors thank the technicians of the Department of Laboratory Medicine for CSF analysis. This work was supported by a grant from Zon-MW Innovational Research (number 917.46.331, "Vidi program", to MMV)

Authors' disclosures available online (http://www.jalz.com/disclosures/view.php?id=389).

\section{REFERENCES}

[1] De Jong D, Kremer BPH, Olde Rikkert MGM, Verbeek MM (2007) Current state and future directions of neurochemical biomarkers for Alzheimer's disease. Clin Chem Lab Med 45, 1421-1434.

[2] World Health Organization (1998) Global surveillance, diagnosis, and therapy of human transmissible spongiform encephalopathies: report of WHO consultation. WHO, Geneva 1998. WHO/EMC/ZDI/98.9., http://www.who.int/topics/ creutzfeldtjakob_syndrome/en/

[3] Otto M, Stein H, Szudra A, Zerr I, Bodemer M, Gefeller O, Poser S, Kretzschmar HA, Mäder M, Weber T (1997) S-100 protein concentration in the cerebrospinal fluid of patients with Creutzfeldt-Jakob disease. J Neurol 244, 566-570.

[4] Sanchez-Juan P, Green A, Ladogana A, Cuadrado-Corrales N, Sáanchez-Valle R, Mitrováa E, Stoeck K, Sklaviadis T, Kulczycki J, Hess K, Bodemer M, Slivarichová D, Saiz A, Calero M, Ingrosso L, Knight R, Janssens AC, van Duijn CM, Zerr I (2006) CSF tests in the differential diagnosis of Creutzfeldt-Jakob disease. Neurology 67, 637-643.

[5] Rosengren LE, Karlsson JE, Sjögren M, Blennow K, Wallin A (1999) Neurofilament protein levels are increased in dementia. Neurology 52, 1090-1093.

[6] Sjögren M, Rosengren L, Minthon L, Davidsson P, Blennow $\mathrm{K}$, Wallin A (2000) Cytoskeleton proteins in CSF distinguish frontotemopral dementia from AD. Neurology 54, 1960-1964.

[7] De Jong D, Jansen RW, Pijnenburg YA, van Geel WJ, Borm GF, Kremer BP, Verbeek MM (2007) CSF neurofilament proteins in the differential diagnosis of dementia. J Neurol Neurosurg Psychiatry 78, 936-938.

[8] Liberski PP, Budka H (1999) Neuroaxonal pathology in Creutzfeldt-Jacob disease. Acta Neuropathologica 97, 329334.

[9] Dubois B, Feldman HH, Jacova C, Dekosky ST, BarbergerGateau P, Cummings J, Delacourte A, Galasko D, Gauthier S, Jicha G, Meguro K, O'brien J, Pasquier F, Robert P, Rossor M, Salloway S, Stern Y, Visser PJ, Scheltens P (2007) Research criteria for the diagnosis of Alzheimer's disease: revising the NINCDS-ADRDA criteria. Lancet Neurol 6, 734-746.
[10] Van Everbroeck B, Dobbeleir I, De Waele M, De Deyn P, Martin JJ, Cras P (2004) Differential diagnosis of 201 possible Creutzfeldt-Jakob disease patients. J Neurol 251, 298-304.

[11] Van Geel WJA, de Reus HPM, Nijzing H, Verbeek MM, Vos PE, Lamers KJB (2002) Measurement of Glial Fibrillary Acidic Protein in blood: an analytical method. Clin Chim Acta 326, 151-154.

[12] Van Geel WJA, Rosengren LR, Verbeek MM (2005) An enzyme immunoassay to quantify neurofilament light chain in cerebrospinal fluid. J Immunol Methods 296, 179-185.

[13] Abdo WF, Bloem BR, Van Geel WJ, Esselink RA, Verbeek MM (2007) CSF neurofilament light chain and tau differentiate multiple system atrophy from Parkinson's disease. Neurobiol Aging 28, 742-747.

[14] Petzold A (2005) Neurofilament phosphoforms: surrogate markers for axonal injury, degeneration and loss. J Neurol Sci 233, 283-298.

[15] Sjögren M, Blomberg M, Jonsson M, Wahlund LO, Edman A, Lind K, Rosengren L, Blennow K, Wallin A (2001) Neurofilament protein in cerebrospinal fluid: a marker of white matter changes. J Neurosci Res 66, 510-516.

[16] Otto M, Wiltfang J, Cepek L, Neumann M, Mollenhauer B, Steinacker P, Ciesielczyk B, Schulz-Schaeffer W, Kretzschmar HA, Poser S (2002) Tau protein and 14-3-3 protein in the differential diagnosis of Creutzfeldt-Jacob disease. Neurology 58, 192-197.

[17] Nagele RG, Venkataraman V, Imaki H, Wang KC, Wegiel J (2004) Contribution of glial cells to the development of amyloid plaques in AD. Neurobiol Aging 25, 663-674.

[18] Aoki T, Kobayashi K, Isaki K (1999) Microglial and astrocytic change in brains of Creutzfeldt-Jakob disease: an immunocytochemical and quantitative study. Clin Neuropathol 18, 51-60.

[19] Jesse S, Steinacker P, Cepek L, von Arnim C, Tumani H, Lehnert S, Kretzschmar HA, Baier M, Otto M (2009) Glial fibrillary acidic protein and protein S100B: different concentration pattern of glial proteins in cerebrospinal fluid of patients with Alzheimer's disease and Creutzfeldt-Jakob disease. J Alzheimers Dis 17, 541-551.

[20] Van Everbroeck B, Quoilin S, Boons J, Martin JJ, Cras P (2003) A prospective study of CSF markers in 250 patients with possible Creutzfeldt-Jakob disease. J Neurol Neurosurg Psychiatry 74, 1210-1214.

[21] Ladogana A, Sanchez-Juan P, Mitrová E, Green A, CuadradoCorrales N, Sánchez-Valle R, Koscova S, Aguzzi A, Sklaviadis T, Kulczycki J, Gawinecka J, Saiz A, Calero M, van Duijn CM, Pocchiari M, Knight R, Zerr I (2009) Cerebrospinal fluid biomarkers in human genetic transmissible spongiform encephalopathies. J Neurol 256, 1620-1628.

[22] Bahl JM, Heegaard NH, Falkenhorst G, Laursen H, Høgenhaven H, Mølbak K, Jespersgaard C, Hougs L, Waldemar G, Johannsen P, Christiansen M (2009) The diagnostic efficiency of biomarkers in sporadic Creutzfeldt-Jakob disease compared to Alzheimer's disease. Neurobiol Aging 30, 1834-1841.

[23] Beaudry P, Cohen P, Brandel JP, Delasnerie-Lauprêtre N, Richard S, Launay JM, Laplanche JL (1999) 14-3-3 protein, neuron-specific enolase, and S-100 protein in cerebrospinal fluid of patients with Creutzfeldt-Jakob disease. Dement Geriatr Cogn Disord 10, 40-46.

[24] Collins S, Boyd A, Fletcher A, Gonzales M, McLean CA, Byron K, Masters CL (2000) Creutzfeldt-Jakob disease: diagnostic utility of 14-3-3 protein immunodetection in cerebrospinal fluid. J Clin Neurosci 7, 203-208. 
[25] Pennington C, Chohan G, Mackenzie J, Andrews M, Will R, Knight R, Green A (2009) The role of cerebrospinal fluid proteins as early diagnostic markers for sporadic CreutzfeldtJakob disease. Neurosci Lett 455, 56-59.

[26] Zerr I, Pocchiari M, Collins S, Brandel JP, de Pedro Cuesta J, Knight RS, Bernheimer H, Cardone F, Delasnerie-Laupiêtre N, Cuadrado Corrales N, Ladogana A, Bodemer M, Fletcher A, Awan T, Ruiz Bremón A, Budka H, Laplanche JL, Will RG, Poser S (2000) Analysis of EEG and CSF 14-3-3 proteins as aids to the diagnosis of Creutzfeldt-Jakob disease. Neurology $\mathbf{5 5}, 811-815$.

[27] Lemstra AW, van Meegen MT, Vreyling JP, Meijerink PH, Jansen GH, Bulk S, Baas F, van Gool WA (2000) 14-3-3 testing in diagnosing Creutzfeldt-Jakob disease: a prospective study in 112 patients. Neurology 55, 514-516.
[28] Zerr I, Bodemer M, Räcker S, Grosche S, Poser S, Kretzschmar HA, Weber T (1995) Cerebrospinal fluid concentration of neuron-specific enolase in diagnosis of CreutzfeldtJakob disease. Lancet 345, 1609-1610.

[29] Jansen C, Schuur M, Spliet WGM, van Gool WA, van Duijn CM, Rozemuller AJM (2009) Elf jaar obducties wegens de ziekte van Creutzfeldt-Jakob in Nederland. Ned Tijdschr Geneeskd 153, A172.

[30] Sanchez-Juan P, Sánchez-Valle R, Green A, Ladogana A, Cuadrado-Corrales N, Mitrová E, Stoeck K, Sklaviadis T, Kulczycki J, Hess K, Krasnianski A, Equestre M, Slivarichová D, Saiz A, Calero M, Pocchiari M, Knight R, van Duijn CM, Zerr I (2007) Influence of timing on CSF tests value for CreutzfeldtJakob disease diagnosis. J Neurol 254, 901-906. 